\title{
Terapia com laser 670nm no tratamento da DPOC experimental em ratos
}

\section{0nm laser therapy in treatment of COPD in experimental rats}

\author{
Wellington dos Santos Alves'; Geraldo Cardoso de Oliveira Júnior²; Luanna Anielle Evangelista \\ do Santo2; Márcia Rejane da Silva Beserra²; Flóvio Aimbire Soares de Carvalho³ \\ ${ }^{1}$ Mestre em Bioengenharia e Professor de Biologia celular e Genética para os Cursos de Fisioterapia - FSA e da UESPI. Teresina PI - Brasil. \\ ${ }^{2}$ Acadêmicos do curso de bacharelado em Fisioterapia - FSA. Teresina, PI - Brasil. \\ ${ }^{3}$ Doutor em Engenharia Biomédica e Professor - Instituto de Ciência e Tecnologia - IC\&T; Universidade Federal de São Paulo - \\ UniFesp. São José dos Campos, SP - Brasil. \\ Endereço para correspondência \\ Wellington dos Santos Alves \\ Rua Primeiro de maio, 2450 - Cond. Barcelona, Bl-E Ap.304 Bairro Aeroporto \\ 64002-510 - Teresina, PI [Brasil] \\ wellingtonsa74@yahoo.com.br
}

\begin{abstract}
Resumo
Introdução: DPOC é uma enfermidade respiratória com manifestações sistêmicas que se caracteriza pela presença de obstrução crônica do fluxo aéreo, associada a uma resposta inflamatória. Objetivo: Verificar o efeito da laserterapia de laser $670 \mathrm{~nm}$, no tratamento da inflamação pulmonar induzida em ratos. Metodologia: 30 ratos foram divididos em três grupos, dos quais dois foram expostos à fumaça de cigarro durante 45 dias e um deles tratados com laser 670nm. Para análise dos resultados foram realizados LBA e ELISA. Resultados: Os resultados foram submetidos à análise de variância (ANOVA), seguida do teste Newman- Keuls para amostras não pareadas. A análise do LBA demonstrou um aumento altamente significativo no número de neutrófilos no grupo DPOC. O grupo tratado, quando comparado ao grupo DPOC, evidenciou uma diminuição significativa no número de neutrófilos. Para o resultado do ELISA, houve queda altamente significativa de TNF- $\alpha$, quando tratado com laser $670 \mathrm{~nm}$, e significativa de MIP-2 e IL- $\beta$. Conclusão: Verifica-se que a ação do laser de $670 \mathrm{~nm}$ pode atenuar o processo inflamatório induzido.
\end{abstract}

Descritores: DPOC; Inflamação pulmonar; Laser; Neutrófilos.

\begin{abstract}
Introduction: COPD is a respiratory illness with systemic manifestations, characterized by the presence of chronic airflow obstruction associated with an inflammatory response. Objective: To investigate the effect of laser 670nm laser in the treatment of pulmonary inflammation induced in mice. Methods: 30 rats were divided into three groups, two of which were exposed to cigarette smoke for 45 days and one treated with $670 \mathrm{~nm}$ laser. For data analysis and ELISA were performed BAL. Results: Results were subjected to analysis of variance (ANOVA) followed by Newman-Keuls test for unpaired samples. BAL analysis showed a highly significant increase in neutrophils in the COPD group. The treated group compared with the COPD group showed a significant decrease in neutrophils. For the result of the ELISA results were highly significant decrease of TNF- $\alpha$ when treated with $670 \mathrm{~nm}$ laser, and the significant MIP-2 and IL-1 $\beta$. Conclusion: It appears that the action of the $670 \mathrm{~nm}$ laser can attenuate the inflammatory process induced.
\end{abstract}

Key words: COPD;Lasers;Lung inflammation;Neutrophils. 


\section{Introdução}

O Consenso da Sociedade Brasileira de Pneumologia e Tisiologia ${ }^{1}$ (SBPT), publicado em 2004, definiu a doença pulmonar obstrutiva crônica (DPOC) como uma enfermidade respiratória previsível e tratável, com manifestações sistêmicas e que se caracteriza pela presença de obstrução crônica no fluxo aéreo, que não é totalmente reversível, e associada a uma resposta inflamatória anormal à inalação de fumaça de cigarro e outras partículas e gases tóxicos. A DPOC é uma das principais causas de morbidade e mortalidade em todo o mundo. Muitas pessoas sofrem dessa doença há vários anos $\mathrm{e}$ morrem prematuramente a partir dela ou de suas complicações ${ }^{2}$.

A DPOC também está associada aos efeitos sistêmicos, tais como a inflamação sistêmica e a disfunção muscular esquelética ${ }^{3}$. A evidência de inflamação sistêmica inclui a presença de estresse oxidativo sistêmico, concentrações anormais de citocinas circulantes e ativação das células inflamatórias ${ }^{4}$. A evidência da disfunção muscular esquelética inclui a perda progressiva de massa muscular esquelética e a presença de várias anomalias bioenergéticas ${ }^{5}$. Tais efeitos sistêmicos acarretam consequências clínicas importantes, pois contribuem para a limitação da capacidade física do paciente e, dessa forma, para o declínio da sua saúde.

$\mathrm{Na}$ conceituação de DPOC, revisada em Meneses ${ }^{6}$, está implícita uma resposta inflamatória aumentada, cujas características acometem vários órgãos e sistemas, principalmente o respiratório. O processo inflamatório crônico pode produzir alterações dos brônquios (bronquite crônica) e bronquíolos (bronquiolite respiratória), e também causar destruição do parênquima pulmonar (enfisema pulmonar), com a consequente redução de sua elasticidade. A presença dessas alterações é variável em cada sujeito e determinam os sintomas da enfermidade.

Todas as alterações promovidas pela inflamação, aumento da produção de muco, contração da musculatura lisa das vias aéreas e destruição alveolar levam à obstrução de vias aéreas, inadequação da relação ventilação-perfusão, hiperinsuflação pulmonar estática e dinâmica e são responsáveis pelo aparecimento de sintomas relacionados à manifestação clínica da enfermidade. As principais células inflamatórias envolvidas na fisiopatologia da DPOC são os neutrófilos, macrófagos e linfócitos CD8. Os mediadores inflamatórios mais envolvidos nesse processo são fator de necrose tumoral alfa (TNF- $\alpha$ ), leucotrieno B4 (LTB4) e interleucina 8 (IL-8). Esse aspecto fisiopatológico é muito importante na diferenciação do diagnóstico entre DPOC e asma, pois são representados por células e mediadores inflamatórios distintos ${ }^{4,7,8}$.

Alguns estudos demonstram diversos efeitos biológicos associados à terapia com laser de baixa potência, tais como crescimento celular estimulado (tecidos conjuntivos, tendinoso e ósseo), reparação celular (aceleração nas células nervosas), efeito anti-inflamatório (redução da capacidade dos linfócitos em reagir a estímulos antigênicos), entre outros. Estudos bibliográficos apresentam uma série de autores que demonstram a ação do LBP em patologias que afetam regiões de tendão, auxiliam na cicatrização de queimaduras, na hiperreatividade da traqueia e poucos trabalhos na utilização dessa terapia nas patologias que acometem as vias aéreas e o pulmão com a redução do processo inflamatório e diminuição do mecanismo de migração de células. Do mesmo modo, na literatura há uma série de publicações que apontam a indução de enfisema pulmonar em ratos e cobaias pela inalação passiva de fumaça de cigarros e a demonstração de células e mediadores sistêmicos de inflamação que participam desse processo. Diante desse aspecto, esse trabalho visa inserir a laserterapia de baixa potência entre as possíveis modalidades terapêuticas para a redução da inflamação pulmonar, provocada pelo uso do cigarro, promovendo essa inflamação em ratos e, posteriormente, o seu tratamento com laser de 670nm. Em seguida, comparam-se os resultados dos diversos grupos de animais, 
no que diz respeito à presença de neutrófilos e mediadores inflamatórios.

\section{Metodologia}

Após aprovação pelo Comitê de Ética e Pesquisa da Univap, protocolo A013CEP/2009, e pelo Comitê de Ética em Pesquisa com Animais da Faculdade Santo Agostinho, protocolo $\mathrm{n}^{\circ}$ 190/09, 30 ratos, da espécie Rattus novergicus da variedade wistar, todos machos, e com peso em torno de $220 \mathrm{~g}$ a $250 \mathrm{~g}$ foram selecionados do biotério da Faculdade Santo Agostinho, em Teresina e acondicionados em gaiolas forradas com palha, sendo fornecido ração e água ad libitum. Os animais estavam divididos em três grupos com 10 animais em cada.

No grupo A, chamado controle, os animais respiravam o ar ambiente, sem nenhum tipo de poluente ou fumaça de cigarro. No grupo B, denominado DPOC, os animais em pares inalavam, de forma passiva, doses de fumaça de dois cigarros comerciais (alcatrão 13,0 mg, nicotina $1,10 \mathrm{mg}$, monóxido de carbono $10 \mathrm{mg}$ ) em sete sessões diárias de 10 minutos, totalizando 14 cigarros por dia para cada dupla. No grupo C, chamado laser 670 , os animais sofriam as mesmas aplicações de cigarro que o grupo B e após os 45 dias de inalação foram tratados por 15 dias com laser de diodo Índio Gálio Alumínio Fósforo (InGaAlP) de 670nm (Laser Bioset modelo physiolux dual, $30 \mathrm{~W}$ de potência e comprimento de onda de $670 \mathrm{~nm}$, densidade de energia de $\left.7 \mathrm{~J} / \mathrm{cm}^{2}\right)$.

Os animais eram colocados aos pares em caixas de madeira (figura 1), com dimensões de $40 \mathrm{~cm}$ de comprimento por $25 \mathrm{~cm}$ de altura e $30 \mathrm{~cm}$ de largura, dimensões observadas em Valença e Porto $^{9}$, com modificações para nosso trabalho. A caixa apresentava um orifício nas faces laterais de $30 \mathrm{~cm}$, onde foi colocado um cigarro de cada lado, protegido com tela de náilon para não agredir o animal, e quatro orifícios superiores, sendo dois em cada tampa para promover uma pequena circulação de ar no meio. A aplicação de cigarros durou 45 dias, considerando-se seis dias por semana. Era um total de cinco caixas nas quais um grupo passava por uma sessão de inalação, vindo outro em seguida, de tal modo que o intervalo de inalação entre o B e C marcava 15 minutos.

Para o tratamento, os animais do laser 670 foram anestesiados inicialmente com Ketamina -100 mg/kg, im (DOPALEN INJETÁVEL- 10 ml- Vetbrands $\left.{ }^{\circledR}\right)$ e Xilazina $-20 \mathrm{mg} / \mathrm{kg}$, im (ANASEDAN INJETÁVEL -10 ml- Vetbrands®) para tricotomia. Durante os dias de recebimento de laser, as aplicações foram feitas em três pontos, um na região da carina, e outra em cada hemitórax, fechando uma forma triangular. Cada animal recebia uma dose por dia, com duração de $21 \mathrm{~s}$ em cada ponto, e calculada pelo próprio aparelho, a uma potência de $30 \mathrm{~mW}$ e densidade de energia de 7 J. O tempo de exposição foi calculado com a multiplicação da densidade $\left(\mathrm{J} / \mathrm{cm}^{2}\right)$ pela área $\left(\mathrm{cm}^{2}\right)$, dividida pela potência de emissão (W), a energia deve ser medida e calibrada para não sobrecarregar ou não ser ineficiente no meio vivo. Esse procedimento é automático hoje nos aparelhos modernos por meio de seletores de densidade de potência, desde que seja fornecida a dose indicada.
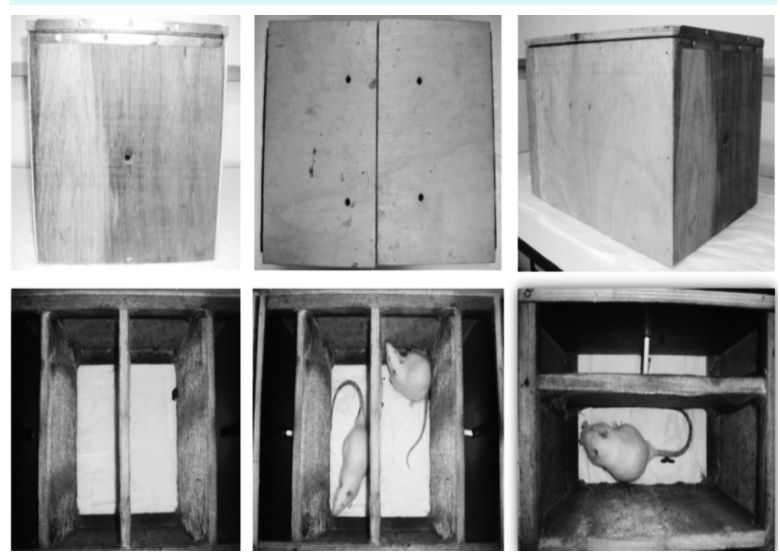

Figura l: Câmara para inalação do cigarro

O grupo B foi sacrificado com dose de $300 \mathrm{mg} / \mathrm{Kg}$ de tiopental para a preparação de lâminas do LBA. Em cinco dos animais, o pulmão esquerdo foi direcionado para o exame de Elisa 
para identificação de marcadores inflamatórios, como interleucinas e $\alpha$-TNF. Os outros cinco foram direcionados ao lavado bronco alveolar para a contagem de neutrófilos. Nesse procedimento, os animais foram anestesiados, como já descrito anteriormente, e a seguir foi feita uma incisão longitudinal na região cérvico-ventral, assim a traqueia ficou exposta e canulada e foi acoplada a uma das suas entradas uma cânula; a uma segunda entrada, uma seringa contendo $20 \mathrm{ml}$ de PBS (Solução Salina Fosfatada Tamponada) que foi ejetada no pulmão, aplicada uma massagem local e depois aspirados $10 \mathrm{ml}$ de secreção pulmonar. O Lavado Broncoalveolar(LBA) obtido foi centrifugado por 10 minutos a $1500 \mathrm{rpm}$. O sobrenadante desprezado e o botão celular ressuspenso em $1 \mathrm{ml}$ de PBS. O esfregaço foi corado com o método May Grnwald - Giensa e as lâminas para a contagem das células totais realizada em câmara de Neubauer. O grupo C, após os 15 dias de tratamento com o laser, também passou pelos mesmos procedimentos de preparo histológico, teste Elisa e LBA já descritos para o grupo $\mathrm{B}$, o mesmo acontecendo com o grupo controle.

Os resultados foram submetidos à análise de variância (ANOVA) e, em seguida, ao teste Newman- Keuls para amostras não pareadas. As análises estatísticas foram conduzidas utilizando o GraphPad Software V. 4, GraphPad InstatTM (2005). Os resultados foram expressos como a média \pm erro padrão da média (EPM). Valores de $p<0,05$ foram considerados significativos.

\section{Resultado e discussão}

Estudos com modelos animais, em especial ratos ou camundongos, mostram que a exposição à fumaça de cigarros, de forma passiva, por menor tempo possível já é capaz de ativar células inflamatórias e mediadores que podem acarretar inflamações pulmonares relacionadas ao enfisema ${ }^{9-11}$. Essa inflamação é caracterizada pela presença de macrófagos, neutrófilos, linfócitos $\mathrm{CD} 8+$, associada à alteração do número de fibras colágenas e elásticas e ao aumento do diâmetro dos alvéolos pulmonares, contribuindo com o ganho do espaço morto fisiológico e com a diminuição da capacidade respiratória dos indivíduos ${ }^{4}$. Esse processo tem a participação de substâncias químicas, como os mediadores TNF- $\alpha$, TGF- $\beta$, IL- $1 \beta$ e outros que promovem uma cadeia inflamatória degradando o parênquima pulmonar ou aumentando a secreção nessa área, provocando a injúria respiratória ${ }^{11,12}$.

Os neutrófilos, uma das principais células presentes na inflamação pulmonar, são responsáveis pela produção de serinas proteases, enzimas proteolíticas neutrofílicas que apresentam várias atividades, como a degradação de fibras elásticas no tecido pulmonar, sendo uma das poucas enzimas humanas capazes de realizar essa destruição ${ }^{13}$.

Corroborando com esses dados, essa pesquisa com ratos Wistar que inalaram durante 45 dias fumaça de 14 cigarros por dia, também mostra os mesmos resultados em termos de células e da presença de mediadores inflamatórios do grupo das citocinas e quimiocinas, e, ao mesmo tempo, o efeito anti-inflamatório do laser de baixa potência que foi utilizado como mecanismo de tratamento não medicamentoso e não invasivo, pois após o período de inalação, os animais tratados por 15 dias, com uma aplicação diária de laser em três pontos, uma à nível da carina, e outra em cada hemitórax, promoveu uma redução considerável nas células do tipo neutrófilos no LBA e nos mediadores testados pelo ELISA, como mostra nos gráficos abaixo.

Dados do LBA (figura 2) demonstraram um aumento altamente significativo $(p<0,001)$ no número de neutrófilos no grupo DPOC quando comparado ao grupo controle. Resultados semelhantes foram encontrados em Shapiro ${ }^{14}$ ao realizar um estudo utilizando 26 camundongos com deficiência da elastase neutrofílica (NE-), 20 com presença de elastase neutrofílica (NE+) da linhagem do tipo selvagem, 12 com elastase macrofágica (MMP12) e 24 que não foram expostos a fumaça de cigarro. Os camundongos NE-, $\mathrm{NE}+\mathrm{e}$ MMP12 foram submetidos a dois cigarros sem filtro por dia, sendo seis dias por semana, 
durante seis meses. Por meio de avaliação feita pelo LBA, observou-se que os camundongos da linhagem do tipo selvagem (NE+) tiveram um aumento cinco vezes maior no número de neutrófilos quando comparados com os camundongos NE- expostos a fumaça de cigarro; com relação aos macrófagos, os números de linha de base não foram afetados pela falta genética de NE.

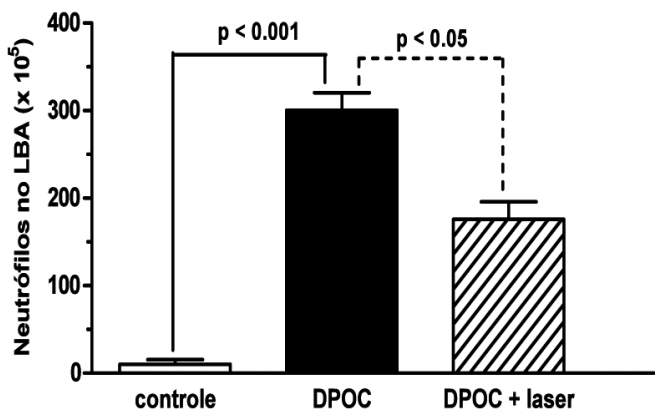

Figura 2: Neutrófilos no lavado broncoalveolar. Mostra a diminuição de neutrófilos no grupo tratado com laser em relação ao grupo DPOC e, ao mesmo tempo, a existência da inflamação com o aumento de neutrófilos do grupo DPOC em relação ao controle

Verifica-se que, em relação ao controle, o número de neutrófilos é bem mais abundante no DPOC, representando $350 \times 10^{5}$, o que indica a presença de inflamação, e no grupo tratado com laser esse valor reduz para cerca de $180 \times 10^{5}$, mostrando que o laser exerceu função antiinflamatória ao proporcionar a redução dessas células, pois entre os grupos DPOC e DPOC + laser, a redução foi significativa $(\mathrm{p}<0,05)$. De acordo com Tarantino ${ }^{4}$, essas células são as mais encontradas no LBA de pacientes com DPOC e, como mostra Rufino ${ }^{15}$, em seus trabalhos, ocorre um aumento considerável dessas células em contagem de células do escarro em pacientes com DPOC em relação aos indivíduos tabagistas sem DPOC e não tabagistas.

Os neutrófilos estão presentes juntamente com o mediador inflamatório TNF- $\alpha$, que como mostra a figura 4, também está abundante no grupo B e acompanha a queda de neutrófilos no grupo C, tratado com laser 670nm. Essas células estão implicadas na liberação de citocinas inflamatórias, mediadores lipídicos e enzimas capazes de promover dano tecidual ${ }^{16}$, além disso, podem promover hipersecreção de muco e proliferação de glândulas mucosas ${ }^{17}$. O TNF- $\alpha$ é produzido por macrófagos e ou células do epitélio respiratório que proporcionam uma comunicação celular por meio de sinais químicos. Esse mediador é uma substância da categoria das monocinas e sua secreção pelos macrófagos pode ser ativada por vários estímulos, exercendo atividades, tais como a proliferação, diferenciação e apoptose celular, o que provoca, no caso da DPOC, a destruição do parênquima, estimula a liberação de colagenases e aumenta a expressão de moléculas de adesão encontradas na DPOC, provocando o extravasamento leucocitário que atrai neutrófilos que, embora apresentem um menor diâmetro em relação aos poros do capilar sanguíneo pulmonar, conseguem sair e atuar na parede pulmonar provocando inflamação, especialmente na DPOC grave.

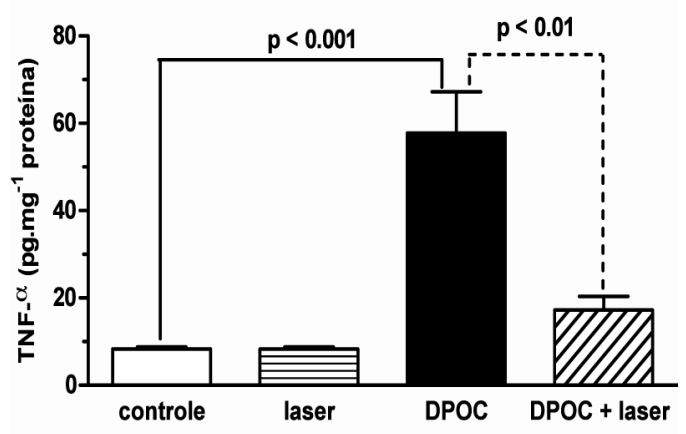

Figura 3: Concentração de TNF- $\alpha$ no infiltrado pulmonar após a análise pelo teste de ELISA. Verifica-se uma redução na concentração do mediador inflamatório presente no grupo DPOC quando tratado com laser a valores quase próximos ao do controle

Conforme explica $\mathrm{Karu}^{18}$ em suas publicações, o laser atua não fornecendo calor, mas sim promovendo uma bioestimulação nas organelas celulares, que ocasiona a síntese de compostos protéicos, como os anti-inflamatórios que inibem a ação dos IL- $1 \beta$ e TNF- $\alpha$, 
promovendo efeitos sistêmicos, dessa forma a laserterapia de baixa potência, além de ter função analgésica, também é anti-inflamatória, pois interrompe a comunicação celular que, por quimiotaxia, atrai os mediadores para o local da fisiopatologia respiratória.

Verifica-se também na figura 5 que os níveis de MIP2 estão elevados nos animais DPOC, e muito reduzidos no controle e laser, havendo uma leve redução no DPOC+laser. Essa quimiocina tem sua expressão estimulada pela ação do TNF- $\alpha$ que, como visto antes na figura 3, tem um nível elevado no grupo DPOC e reduzido nos animais que foram tratados com laser, mostrando que a reação em cascata que desencadeia na inflamação também tem como efeito a queda quando aplica-se o laser, reduzindo os agentes inflamatórios, desde o número de células, até a diminuição de todos os mediadores que participam do processo, como também mostrado na figura 5 em relação à IL- $1 \beta$.

Aimbire $^{19}$, em seus estudos, já demonstrou que o laser reduz a atividade do TNF- $\alpha$, na inflamação pulmonar, inclusive aumentando a capacidade mecânica do diafragma com a redução desse composto no músculo respiratório em ratos. Esse trabalho mostra também a redução de outros mediadores. Alguns artigos mostram terapias atuais e futuras para o tratamento de enfisema pulmonar, entre elas há a utilização de receptores para TNF- $\alpha$, que mesmo em ratos

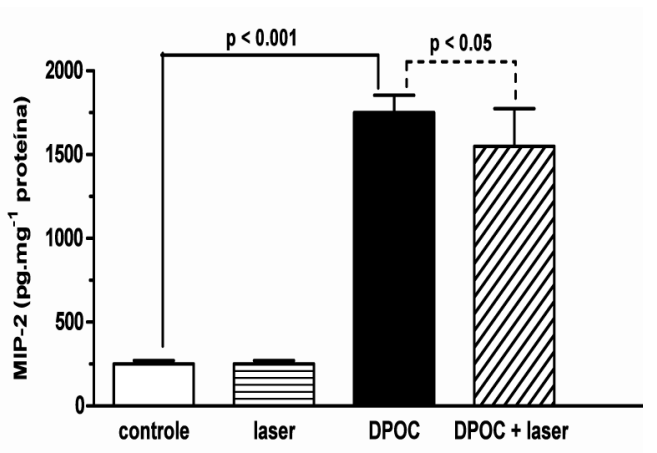

Figura 4: Ação do laser na redução do MIP-2. Hó uma queda discreta promovida pela ação do laser que diminui a ação do TNF- $\alpha$, um dos principais estimuladores da secreção de MIP-2 com exposição à fumaça de cigarros, diminuem o quadro inflamatório, efeito também encontrado nessa pesquisa quando da utilização do laser $670 \mathrm{~nm}$, o que evidencia a laserterapia como uma perspectiva de tratamento futuro, pois alguns trabalhos sugerem que a interrupção dos receptores específicos para os mediadores podem reduzir a cascata inflamatória, e o laser atua, como analgésico e também como anti-inflamatório nessa pela interrupção da comunicação celular.

Vários autores ${ }^{20-25}$, verificaram importantes efeitos anti-inflamatórios e analgésicos da terapia laser de baixa potência . A terapia com esse tipo de laser pode ser efetiva, ao mediar os sintomas do processo inflamatório, por meio da estabilização da membrana celular pela normalização das concentrações de cálcio, sódio e potássio, contribuindo com a reparação celular; com a vasodilatação, aumentando o transporte de oxigênio e nutrientes para as células danificadas e facilitando a reparação e remoção dos restos celulares; acelerando a atividade dos leucócitos; aumentando a síntese de prostaglandina em razão da conversão das prostaglandinas PGG2 e PGH2 periosídeos em prostaglandinas PGI2, que possuem ação vasodilatadora e anti-inflamatória, reduzindo a interleucina I, aumentando a resposta dos linfócitos, o que afeta beneficamente toda a resposta linfática, estimulando a angiogênese de capilares linfáticos e sanguíneos por induzir o aumento de

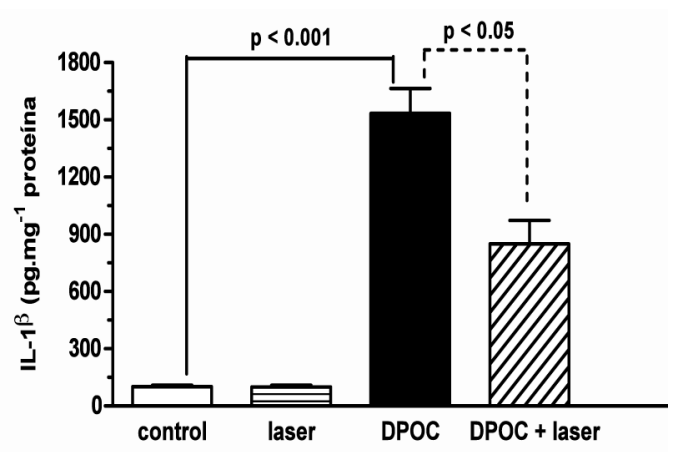

Figura 5: Mostra a concentração elevada de IL-1 $\beta$ no grupo exposto a fumaça de cigarro, o que ocasiona inflamação pulmonar, havendo uma redução do mediador inflamatório no grupo tratado com laser $670 \mathrm{~nm}$ 
óxido nítrico e de fatores do crescimento que contribuem com esse processo, levando a um aumento na capacidade de aderência celular e, assim, da reparação tecidual ${ }^{26,27 .}$

\section{Conclusão}

Com base nas análises realizadas, verificase que a inflamação pulmonar pela inalação do cigarro, após ser comprovada, pôde ser tratada com a laserterapia de baixa potência, o que atenuou o processo inflamatório, uma vez que reduziu a presença de neutrófilos e diminuiu a concentração de mediadores inflamatórios determinantes do enfisema pulmonar, agindo diretamente na reação em cascata da cessação do processo inflamatório. Portanto, nota-se a necessidade de novos estudos que possam corroborar com esses resultados, podendo, futuramente, fundamentar a utilização da LBP no tratamento de pacientes com DPOC, talvez não como um procedimento que leve a sua cura, mas que proporcione uma nova modalidade de combate a essa doença respiratória e proporcione uma melhora na qualidade de vida do seu portador, pois conforme Aimbire ${ }^{28}$, apesar dos efeitos anti-inflamatórios já observados pela TLBP, esta provavelmente não substituirá os medicamentos utilizados hoje no tratamento de afecções pulmonares agudas, apesar das ações já comprovadas em experimentos que o laser inibe estímulos quiomiotáxicos na fase inicial do processo inflamatório.

\section{Referências}

1. II Consenso Brasileiro sobre Doença Pulmonar Obstrutiva Crônica - DPOC - 2004. Jornal Brasileiro de Pneumologia, 5 nov.2004;30.

2. Nascimento AO, Jardim JR. Epidemiologia, impacto e tratamento da Doença Pulmonar Obstrutiva Crônica (DPOC) no Brasil. Revista Recime;2003.
3. American Thoracic Society, European Respiratory Society. Skeletal muscle dysfunction in chronic obstructive pulmonary disease. Am J Respir Crit Care Med.1999;159: S1-40.

4. Tara4ntino, A. B. Doenças pulmonares. $7^{\mathrm{a}}$ ed. Rio de Janeiro: Guanabara Koogan; 2002.

5. Silva K.R, Marrana K.T, Marino D.M, Di Lorenzo VAP, Jamami M. et al. Fraqueza muscular esquelética e intolerância ao exercício em pacientes com doença pulmonar obstrutiva crônica. Rev Brasil Fisiot. São Carlos. Maio/jun. 2008;12(3,):169-75.

6. Meneses AMB. Prevalence of chonic obstructive pulmonary disease and associated factors: the platino study in São Paulo, Brazil. Cad Saúde Pública, Rio de Janeiro. set-out/2005;21(5):1565-73.

7. Geraldes L, Todo-Bom A, Loureiro C. Avaliação da inflamação das vias aéreas. Vias aéreas superiores e compartimento broncopulmonar. Revista Portuguesa de Pneumologia. Maio/Junho.2009;15(3).

9. Wright J.L,Churg A. Current concepts in mechanisms of emphysema. Toxicologic Pathology. 2007;35:111-5.

9. Valença SS, Porto LC. Estudo imunohistoquímico do remodelamento pulmonar em camundongos expostos à fumaça de cigarro. Jornal Brasileiro de Pneumologia. 2008;34(10):787-95.

10. Shapiro SD. Animal models for chronic obstructive pulmonary disease. age of klotho and marlboro mice. Am. J. Respir. Cell Mol. Biol.2000;22:4-7.

11. Barnes PJ, Stockley RA. COPD: current therapeutic interventions and future approaches. Eur Resp, 2005;25:1084-1106.

12. Chung KF, AdcockJ.M. Multifaceted mechanisms in COPD: inflammation, immunity, and tissue repair and destruction. Eur Respir. . 2008;31:1334-56. Series "Cells and animal studies in respiratory medicine".

13. Serra HG. et al. Avaliação da concentração de alfa 1-antitripsina e da presença dos alelos S e Z em uma população de indivíduos sintomáticos respiratórios crônicos. J Bras Pneumol conferir. Dez.2008;34(12): 1019-25.

14. Shapiro SD et al. Neutrophil elastase contributes to cigarette smoke-induced emphysema in mice. American Journal of Pathology;2003.

15. Rufino R, Silva JRL. Bases celulares e bioquímicas da doença pulmonar obstrutiva crônica. J Bras Pneumol. 2006;32(3):241-8. 
16. Holz $\mathrm{O}$ et al. Assessing airway inflammation in clinical practice - experience with spontaneous sputum analysis. BMC Pulmonary Medicine;2008.

17. Costa $\mathrm{CH}$, Rufino R, Silva JRL. Células inflamatórias e seus mediadores na patogênese da DPOC. Revista da Associação Médica Brasileira. 2009;55(3):347-54. .

18. Karu TI. Primary and secundary mechanisms of action of visible to near-IR irradiation on cells. Journal of Photobiology B: Biol. 1999;.49:1-17.

19. Aimbire F, Albertine R, Magalhães RG, LopesMartins RAB, Castro Faria Neto HC, Zangaro RA, Chavantes C, Pacheco MT. Effect of LLLT Ga-Al-As $(685 \mathrm{~nm})$ on LPS induced inflammation of the airway and lung in the rat.Lasers Med Sci.2005;20(1):11-20.

20. Guerino MR. et al. Laser treatment of experimentally induced chronic arthritis. Applied Surface Science;2000.

21. Ciconelli KPC. et al. Bioestimulação óssea utilizando laser de baixa densidade de potência diodo semicondutor $830 \mathrm{~nm}$ em caso de micro mini implante. Jornal Brasileiro de Odontologia Clínica;1998.

22. Cecchini RCM, Cecchini SCM. O papel coadjuvante do Nd: YAG laser na endodontia. Caso clínico. Revista da Associação Brasileira de Odontologia Nacional;1993.
23. Jimbo K. et al. Suppressive effects of low-power laser irradiation on bradykinin evoked action potentials in cultured murine dorsal root ganglion cells. Neuroscience Letters;1998.

24. Walker J. Relief from chronic pain by low power laser irradiation. Neuroscience Letters;1983.

25. Bertolini GRF, Nohama, P. Avaliação da conformidade dos equipamentos laser de baixa potência e emissão contínua empregados em Fisioterapia. Fisioterapia em Movimento;2007.

26. Costardi CHZ. et al. Efeito do laser de baixa intensidade (670nm) após contusão muscular em ratos. Fisioterapia em Movimento;2008.

27. Bolognoni L. et al. Effects of low-power $632 \mathrm{~nm}$ radiation (HeNe laser) on a human cell line: influence on adnylnucleotides and cytoskeletal structures. Journal of Photochemistry and Photobiology B: Biology1994.

28. Aimbire FSC, Bjordal JM, Iversen VV, Albertine R, Frigo L, Pacheco MT, Castro Caire Faria Neto HC., Chavantes MC, Labat RM., Lopes-Martins RAB. Low level laser therapy partially restores trachea muscle relaxation response in rats with Tumor Necrosis Factor-a mediated smooth airway muscle dysfunction. Lasers in surgery and medicine.2006;38(8):773-8. 